\title{
Using a Transdiagnostic Perspective to Disrupt White Supremacist Applications of the DSM
}

\author{
Michael R. Riquino \\ Van L. Nguyen \\ Sarah E. Reese \\ Jen Molloy
}

\begin{abstract}
White supremacist applications of the Diagnostic and Statistical Manual of Mental Disorders (DSM) result in the disproportionate labeling of Black, Indigenous, and People of Color as violent or severely mentally ill. Racial diagnostic disparities and misdiagnoses are endemic in social work practice, in part because of the DSM's categorical classification system, which encourages reductive thinking and reinforces implicit racial biases. While courses on psychopathology are common requirements for clinical field placements, the mental health field's reliance on the DSM often contradicts antiracist curricula. In an effort to address this paradox, we utilize pedagogical approaches that seek to critique and deconstruct White Supremacist applications of the DSM while simultaneously preparing students to enter a field that relies so heavily on diagnostic labels. This is done in part by teaching students to shirk the DSM's categorical perspective in favor of a transdiagnostic perspective-identifying symptoms or traits underlying human suffering that occur across diagnostic categories and are informed by macro systems of privilege and oppression. Teaching students to adopt a transdiagnostic perspective may disrupt White Supremacist practices in diagnostics by encouraging an acknowledgement of multisystem factors underlying human suffering without relying on discrete diagnostic categories that are prone to racial interpretations.
\end{abstract}

Keywords: DSM, White supremacy, transdiagnostic perspective

Applications of the Diagnostic and Statistical Manual of Mental Disorders (DSM; American Psychiatric Association, 2013) in clinical social work practice are typically informed by White Supremacist culture, which implicitly and explicitly codifies racism and privileges Whiteness within institutional structures and individual perspectives. This results in the disproportionate labeling of Black, Indigenous, and People of Color (BIPOC) as dangerous or severely mentally ill. BIPOC are more likely to be diagnosed with schizophrenia for symptoms that result in mood disorder diagnoses in their White counterparts (Akinhanmi et al., 2018; Olbert et al., 2018). Children of color are more likely to be diagnosed with oppositional defiant disorder for symptoms that result in attentiondeficit hyperactivity disorder diagnoses in White children (Ballentine, 2019). Such racial diagnostic disparities are endemic in social work practice, in part because of the categorical classification system of the DSM, which encourages reductive thinking, reinforces implicit racial biases in a primarily White workforce, and supports a false dichotomy between micro and macro practice.

While courses on psychopathology are common requirements for clinical social work

Michael R. Riquino, PhD, MSW, Assistant Professor, School of Social Welfare, University of Kansas, Lawrence, KS. Van L. Nguyen, PhD, MSW, Independent Researcher in Salt Lake City, UT. Sarah E. Reese, PhD, MSW, Assistant Professor, and Jen Molloy, PhD, MSW, Assistant Professor, School of Social Work, University of Montana, Missoula, MT. 
field placements, the mental health field's reliance on the DSM often contradicts antiracist curricula encouraged in MSW programs. In an effort to address this paradox, we have incorporated pedagogical approaches that seek to critique and deconstruct White Supremacist applications of the DSM while simultaneously preparing students to enter a field that relies so heavily on diagnostic labels. This is done in part by teaching students to shirk the DSM's categorical perspective in favor of a transdiagnostic perspective (SauerZavala et al., 2017) - that is, identifying symptoms or traits underlying human suffering that occur across diagnostic categories - and then integrating that transdiagnostic perspective with critical and socioecological understandings to assess how suffering is informed by macro systems of privilege and oppression.

Teaching social work students from a transdiagnostic perspective may disrupt White Supremacist practices in diagnostics by encouraging them to acknowledge multisystem factors underlying human suffering without relying on discrete diagnostic categories that are prone to racial interpretations. Antiracist social work practice requires social workers to deeply interrogate their own perspectives and biases, and reflect on how their identities impact the spaces they inhabit. We believe teaching psychopathology from a transdiagnostic perspective supports these processes by encouraging social workers to engage in ongoing critical self-reflection and disrupt institutionalized ways of thinking about diagnostics. In this article, we will explore the extant impact of the DSM on the classification of psychological disorders, examine empirical evidence illustrating White Supremacist applications of the DSM, explore pedagogical approaches we have employed to disrupt White Supremacist applications of the DSM, and discuss the benefits of using a transdiagnostic perspective as it relates to antiracist pedagogy and practice.

\section{The Influence of the DSM on the Classification of Psychological Disorders}

The developmental history of the DSM, as well as the appropriateness of its fit within the social work profession (Lyter \& Lyter, 2012), is rife with controversy given its increasing focus on biomedical and socially decontextualized perspectives of psychological disorders (see Bredström, 2019; La Roche et al., 2015; Whooley, 2014). Despite these controversies, social work practitioners, educators, and researchers have been tasked with utilizing the DSM in their practices while balancing its biomedical approach with strengths-based and socioecological perspectives (Probst, 2013). This requirement is largely because social workers provide the bulk of mental health services in the United States (Kourgiantakis et al., 2019) and "the DSM has become the standard in all facets of mental health, including clinical practice, insurance reimbursement, research designs, epidemiological studies, and public policy" (Whooley, 2014, p. 92). The dominance of the DSM in the mental health field requires examining its influence on the classification of psychological disorders, particularly as it relates to its use of a categorical classification system and its decontextualized conceptualizations of mental distress.

Developers of the third edition of the DSM adopted a categorical classification system of psychological disorders in an effort to increase the reliability of diagnostic classification-meaning, experiences of mental distress were descriptively sorted into seemingly discrete categories defined by lists of signs and symptoms (Riboni \& Belzung, 
2017). As Rodriguez-Seijas et al. (2015) described it, the DSM considers psychological disorders "to be dichotomous - being either absent or present. Diagnostic decisions are made by determining whether a given patient's clinical presentation meets a certain...criterion threshold, wherein each criterion deemed present counts equally toward the diagnosis" (p. 425). This categorical approach has persisted to the current fifth edition of the DSM despite its lack of validity (Rodriguez-Seijas et al., 2015; Whooley, 2014), as well as its disregard for socioecological factors that contextualize the presentation and course of psychological disorders. Reviewers of the DSM-5 attempted to address the issue of decontextualization by extending the DSM's definition of culture and developing its Cultural Formulation Interview. However, Bredström (2019) noted these efforts were insufficient to address the complex socioecological contexts in which psychological disorders develop: "What we have is a manual that acknowledges that culture affects all disorders yet only presents some symptoms as culturally contextualized" (p. 360).

Critics of the DSM-5 have argued transdiagnostic and dimensional approaches address its current limitations (Rodriguez-Seijas et al., 2015; Whooley, 2014). Integrating a dimensional approach into the DSM-5 - that is, assessing symptoms on a spectrum versus using criterion thresholds - was the initial goal of DSM-5 reviewers, as they believed doing so would elucidate biological etiologies of mental distress (Whooley, 2014). Further, such a change would have reinforced the appropriateness of adopting a transdiagnostic perspective in assessment given the substantial overlap in diagnostic categories. Transdiagnostic factors may be less prone to diagnostic bias as they have been found to be relatively invariant across social positionalities (Rodriguez-Seijas et al., 2015). However, as Bredström (2019) remarked, "The suggested move towards a dimensional model does not necessarily solve this [etiology] problem as it seems likely that it will focus on measuring severity and recognizing crosscutting symptoms, rather than locating suffering in its cultural context" (p. 360). Ultimately, the DSM-5 retained a categorical approach to diagnostics while providing recommendations for continued research into the appropriateness of adopting a dimensional approach. Disappointed in this decision, the National Institute of Mental Health developed the Research Domain Criteria (RDoC) Framework to encourage researchers to focus on transdiagnostic etiologies using neuroscience techniques.

Whooley (2014) cautioned that despite RDoC's focus on etiology, "RDoC is poised to accelerate the decontextualizing trend by (1) decoupling research and clinical concerns when constructing its [classification system], (2) proffering a reductionist brain-centric model of mental distress, and (3) privileging research built upon neural imaging technologies" (p. 94). We share this author's and other scholars' concerns regarding both the DSM and RDoC's reinforcement of medicalization and biological reductionism. However, as noted earlier, we also recognize the DSM's dominance in the mental health field and the need for social work students to be intimately familiar with the DSM's classification system in order to effectively operate in the field and disrupt its White Supremacist applications. The pedagogical approaches we espouse in this article align with that aspiration of dimensionalization while positioning suffering in socioecological context and allowing for an interrogation of etiology beyond the individual level; for example, how the social environment becomes internalized within individuals and manifests as symptoms 
or traits of psychological disorders.

\section{Examples of White Supremacist Applications of the DSM}

The sequelae of racial diagnostic disparities can include differential access to services and recommended interventions (e.g., Akinhanmi et al., 2018; Gudiño et al., 2012; Simpson et al., 2007), as well as White Supremacist conceptualizations regarding the etiology of psychological disorders (see Bredström, 2019; La Roche et al., 2015). In this section, we begin by examining diagnostic labels disproportionately applied to BIPOC compared to White individuals (i.e., schizophrenia and oppositional defiant disorder vs. major depressive disorder and attention-deficit hyperactivity disorder, respectively), as well as misdiagnoses that are more common among BIPOC compared to White individuals (i.e., bipolar disorder and posttraumatic stress disorder). We then explore how racial diagnostic disparities can result in decreased access to treatment and resources, and how misdiagnoses can result in delayed or harmful treatments. Finally, we explore the sociocultural impact of labeling BIPOC with diagnoses that are generally perceived as dangerous or severely mentally ill, as well as racist conceptualizations regarding the origin of psychological disorders. Specifically, we discuss the stark differences between conceptualizations of the so-called "war on drugs" that primarily impacted Black individuals in the United States compared to the current "opioid epidemic" and its impact on White individuals in the United States.

\section{Racial Diagnostic Disparities and Misdiagnoses}

Racial disparities in schizophrenia diagnoses, especially among Black adults seeking mental health treatment, have been consistently documented (Aggarwal et al., 2012; Akinhanmi et al., 2018; Eack et al., 2012; Olbert et al., 2018; Schwartz \& Blankenship, 2014). Even when diagnoses such as major depressive disorder or bipolar disorder are warranted, BIPOC individuals are more likely than their White counterparts to be diagnosed with schizophrenia - a diagnosis that is most often treated with intense psychotropic medication regimens and accompanied by significant social stigma. These disparities may be explained by the presentation of genuine symptom differences given the discriminatory and oppressive experiences of BIPOC (Feisthamel \& Schwartz, 2009). However, these disparities can also be explained in part by clinician perceptions of dishonesty when BIPOC clients are describing their experiences of distress (Eack et al., 2012; Olbert et al., 2018). Researchers have found "clinicians may fail to situate the diagnostic encounter in appropriate cultural context and misconstrue normative guardedness as paranoia" (Olbert et al., 2018, p. 105). Although client guardedness makes sense in a socioecological context, a decontextualized approach to diagnostics may amplify clinicians' implicit racial biases and result in the pathologizing of normative behavior (Metzl, 2009).

Decontextualization also shows up in posttraumatic stress disorder diagnostic practices. Although BIPOC are more likely to be diagnosed with posttraumatic stress disorder than White individuals (Carter, 2007; Helms et al., 2012), the DSM narrowly defines trauma and fails to acknowledge the potential for oppression and discrimination to 
be traumatic in the lives of BIPOC (Holmes et al., 2016). In essence, the DSM's definition of trauma as "exposure to actual or threatened death, serious injury, or sexual violence" (American Psychiatric Association, 2013, p. 271) privileges interpersonal forms of violence as warranting attention while ignoring "multigenerational transmission of structural trauma (i.e., poverty, hunger, unemployment, mass incarceration) and historical trauma (i.e., slavery, genocide)" (Holmes et al., 2016, p. 316). This conceptualization reifies the DSM's micro-oriented focus, positioning trauma within interpersonal situations and suggesting an individual's response to trauma is a failure to employ adequate coping strategies. Given BIPOC individuals are more likely to be diagnosed with posttraumatic stress disorder, this decontextualized focus pathologizes their experiences while ignoring potential socioecological sources of suffering.

Racial diagnostic disparities and misdiagnoses are not limited to BIPOC adults. Children of color are also more commonly labeled as having behavioral or severe emotional disturbances (Visser et al., 2016). For example, parents and teachers are more likely to perceive Black children as having attention-deficit hyperactivity disorder compared to White children (Gudiño et al., 2012), and yet Black children are more likely to be diagnosed with oppositional defiant disorder than attention-deficit hyperactivity disorder compared to White children (Ballentine, 2019). These findings highlight the multiplicative impacts of racial bias: behavioral difficulties such as inattention and hyperactivity are disproportionately ascribed to Black children, and then compounded by diagnostic attributions of oppositional defiant disorder rather than attention-deficit hyperactivity disorder. Ballentine (2019) noted that stereotypes and implicit bias "may lead diagnosticians to perceive Black children's behavior as outwardly defiant and as the willful choice of a dangerous child associated with [oppositional defiant disorder], rather than through the biological mechanisms of [attention-deficit hyperactivity disorder]" (p. 7). Such misdiagnoses serve to associate Black children with more extreme behavioral difficulties and potentially lead to misperceptions of future conduct or antisocial behavior problems. Rather than receiving greater access to indicated services or treatment, children of color are at a risk of being referred to school resources officers and juvenile court (Evangelist et al., 2017; Fader et al., 2014; Haight et al., 2016), thereby reinforcing racial and ethnic disparities in the juvenile justice system via the school-to-prison pipeline (Kim et al., 2010; Mallett, 2016).

In addition to reifying harmful stereotypes of BIPOC and children of color, these diagnostic disparities and misdiagnoses can result in disproportionate access to services, delayed intervention, and even harmful treatments. Akinhanmi et al. (2018) described how mistaking bipolar disorder for related diagnoses can delay the application of empiricallysupported psychotherapies or result in prescribing contraindicated medication regimens: "A misdiagnosis of bipolar depression as unipolar major depressive disorder with subsequent antidepressant treatment increases the likelihood of treatment non-response and/or antidepressant-induced mania/mood destabilization, while a misdiagnosis of schizophrenia limits the opportunity for treatment with lithium and/or mood-stabilizing anticonvulsants" (p. 507). Olbert et al. (2018) noted how racial disparities in schizophrenia diagnoses may be related to other racial disparities experienced by Black individuals with schizophrenia, including more frequent hospitalizations, higher antipsychotic doses, 
decreased quality of life, and poorer outcomes following first-episode psychosis. In a review of the burden of major depressive disorder on BIPOC versus White individuals, Kim (2014) found "that when [major depressive disorder] affects minority groups, it is usually untreated and is more severe and disabling compared to non-Hispanic whites" (p. 2). This may be due in part to BIPOC's mistrust of mental health services, including personal experiences of racism from mental health providers who exhibited a lack of cultural sensitivity.

\section{Labeling and the Etiology of Psychological Disorders}

The impact of racial diagnostic disparities and misdiagnoses goes beyond the micro level. Disparities and misdiagnoses contribute to sociocultural constructions of BIPOC as dangerous, violent, or severely mentally ill. As outlined in labeling and identity theories (Burke, 2010; Hogg et al., 1995), these constructions can result in social stigma and perpetuate damaging perceptions of group identities. Over time, racial diagnostic disparities can lead to etiological conceptualizations positioned within group identities instead of the oppressive systems affecting those groups. The disproportionate diagnosing of schizophrenia among Black individuals led to researchers citing Blackness as a risk factor for schizophrenia (Metzl, 2009). When Blackness is used as proxy for schizophrenia risk - that is, when mental health providers are taught that Black individuals are more likely than other racial groups to experience schizophrenia instead of being taught that Black individuals are more likely to be diagnosed with schizophrenia due to sociocultural misperceptions - it reinforces the notion that psychosis exists within Black individuals instead of the systems they are required to navigate. These social constructions have impacted how public policies are shaped in response to psychological disorders affecting different group identities.

Reagan's expansion of Nixon's war on drugs policies disproportionately impacted BIPOC, who were more likely to receive longer prison sentences for using crack cocaine compared to their White counterparts for using powder cocaine (Tonry, 1994). These harsh policies shifted public perception to the belief that certain individuals using drugsnamely, BIPOC and poor individuals - were dangerous, violent, and a threat to the United States (Netherland \& Hansen, 2016; Tonry, 1994). This approach contrasts sharply with current rhetoric surrounding the opioid epidemic, which primarily affects White individuals misusing opioid medications (Netherland \& Hansen, 2016). Rather than criminalizing these behaviors, individuals affected by opioid use have been provided with resources or referred for treatment in order to address their behaviors and needs. Although there is significant stigma surrounding substance use generally and opioid use specifically (Tsai et al., 2019), this stigma disproportionately impacts BIPOC and manifests as criminalization of substance use behaviors, decreased access to treatment and services, and more frequent attributions of personal blame for substance use rather than positioning the etiology of addiction within brain chemistry or a broken medical system (Netherland \& Hansen, 2016). The ways in which the DSM has been weaponized to harm BIPOC and support structures of White Supremacy underscores the need for teaching social work students using pedagogical techniques that disrupt these oppressive practices. 


\section{Pedagogical Approaches to Teaching the DSM}

During our time as social work educators, we have repeatedly encountered an especially troubling pedagogical paradox - that is, balancing the need to critically examine and reject the ways in which the DSM reproduces racism and maintains White Supremacy while simultaneously preparing social work students to function in a field that requires its use. Like Lyter and Lyter (2012), we often wonder at the appropriateness of social workers providing diagnostic labels or if the DSM has any place in the social work profession when it so frequently runs contrary to the profession's values and ethical principles. Until such a determination is made to reject the DSM by the social work profession or the mental health community as a whole, we have endeavored to disrupt White Supremacist applications of the DSM by teaching its content through perspectives that align with social work values and ethical principles. In this section, we discuss our pedagogical approaches to teaching psychopathology as it relates to our utilization of transdiagnostic, critical, and socioecological perspectives. We then discuss the impact of our positionalities on the formulation of this manuscript in an effort to demonstrate the reflexivity we encourage in our students.

\section{Transdiagnostic Perspective}

At its core, utilizing a transdiagnostic perspective involves rejecting the DSM's use of a categorical taxonomy to describe psychological disorders and instead considering underlying symptoms and traits that cut across diagnostic categories and inform human suffering (Fusar-Poli et al., 2019). In their review of the transdiagnostic literature base, Sauer-Zavala et al. (2017) found that conceptualizations of the term transdiagnostic fell into two primary definitions: constructs that were descriptively transdiagnostic and those that were mechanistically transdiagnostic. They remarked, "Whereas the designation 'mechanistically transdiagnostic' implies that the construct in question is causally related to a range of psychopathology, the term 'descriptively transdiagnostic' suggests only that a construct is present in multiple disorders, without regard to how or why" (Sauer-Zavala et al., 2017, p. 129).

Anhedonia - the inability to experience pleasure - is one example of a transdiagnostic construct. Anhedonia is only listed once in the DSM as a diagnostic criterion of major depressive disorder (American Psychiatric Association, 2013), and yet individuals who experience anxiety, engage in substance use, or have trauma histories are also vulnerable to anhedonia, indicating it is not unique to one diagnostic label. Further, systemic factors like oppression and poverty can result in anhedonia, suggesting individual treatment is insufficient to address its etiology. Anhedonia is at least descriptively transdiagnostic, as it appears across diagnostic categories despite its singular inclusion in the DSM. It may also be mechanistically transdiagnostic, as anhedonia may play an explanatory role in the development or maintenance of multiple disorders beyond major depressive disorder, including posttraumatic stress disorder, schizophrenia, social anxiety disorder, substance use disorders, and even chronic pain conditions (Bedwell et al., 2014; Destoop et al., 2019).

Sauer-Zavala et al. (2017) advocate reserving the term transdiagnostic for mechanistic 
constructs, as they believe doing so "may function to better inform treatment development, as strategies can be included that focus on these core deficits rather than targeting what may be more trivial disorder correlates" (p. 129). We recommend teaching students to recognize descriptively transdiagnostic constructs while emphasizing the importance of focusing on mechanistically transdiagnostic constructs and how they can inform goal setting and treatment planning. For example, consider racial trauma and its role in the development of posttraumatic stress disorder. The current conceptualization of posttraumatic stress disorder as outlined in the DSM fails to capture how experiences of discrimination and oppression may result in posttraumatic stress symptoms. As Holmes et al. (2016) note, "The current definition of trauma conceptualizes violence within the cultural zeitgeist as primarily interpersonal and physical, while widely discounting systemic, institutional, and psychological trauma" (p. 315). In mental health settings that require DSM diagnoses for treatment, students would be hindered in their ability to provide helpful or accurate treatment if clients' experiences of racism and subsequent racial trauma do not fit the diagnostic threshold for posttraumatic stress disorder as defined by the DSM.

To overcome this, we teach students to recognize the descriptively transdiagnostic nature of the symptomatology of posttraumatic stress disorder, for example, the symptom cluster negative alterations in cognitions and mood is consistent with symptoms described in mood disorders like major depressive disorder, and the symptoms cluster marked alterations in arousal and reactivity is consistent with symptoms described in anxiety disorders like generalized anxiety disorder. With this knowledge, students can rely on alternative diagnostic labels in order to provide treatment in mental health settings that require DSM diagnoses while recognizing that racial trauma may be mechanistically informing the symptoms their clients are experiencing. Like Holmes et al. (2016), we teach our students to conceptualize trauma in the context of systems of oppression in order to "externalize the sources of symptomology. Not only would this serve to depathologize marginalized groups, but accurately naming the problem (e.g., racism, sexism, heterosexism) is an essential first step to coming up with appropriate solutions (e.g., macrolevel interventions, policy change)" (p. 319). By using this mechanistically transdiagnostic understanding of racial trauma, we can teach students to adjust their treatment approach to address clients' needs versus diagnoses.

Although utilizing a transdiagnostic perspective is a process-based approach to diagnostics, there are several concrete strategies we teach students to use in order to disrupt White supremacist applications of the DSM. When more than one diagnosis can parsimoniously explain a client's symptomatology, we teach students to choose diagnoses based on chronicity (e.g., episodic, non-chronic disorders such as major depressive disorder carry less stigma than schizophrenia) and history of racist applications (e.g., children of color have disproportionately been given oppositional defiant disorder diagnoses when attention-deficit hyperactivity disorder diagnoses were appropriate). When chronic or stigmatized diagnoses are the most parsimonious choice to explain a client's symptomatology, we teach students to provide provisional diagnoses as these are typically accepted by insurance companies. This allows students to conduct additional assessment to determine the appropriateness of their diagnoses. 


\section{Critical and Socioecological Perspectives}

Although our pedagogy is focused on teaching students to identify mechanisms that transcend diagnostic categories, akin to the recommendations of the RDoC Framework described earlier, the similarity ends there. Our ultimate goal is to position transdiagnostic mechanisms within a socioecological framework rather than positioning them solely within individual-level variables, such as RDoC's hyperfocus on brain circuitry. We believe consideration should first be given to the impact of systemic forces that result in maladaptive behaviors and functioning on an individual level. Teaching students to understand the potential impact of $\mathrm{RDoC}$ on future diagnostic research and its sequalaeincluding clinical practice - prepares them to disrupt the further decontextualization RDoC is poised to reify. This pedagogy reinforces the notion of applying critical, antiracist perspectives when consuming research that exclusively relies on behavioral neuroscience techniques to the neglect of macro influences.

To accomplish this, we teach our students to filter their transdiagnostic conceptualizations of psychological disorders through critical and socioecological understandings. This process involves recognizing etiologies both outside of and within individuals (Cerulo, 2010), practicing critical self-reflection when conducting assessments (Atteberry-Ash et al., 2019), and acknowledging the role of intersectionality when meeting with individual clients (Crenshaw, 1991). Further, we promote the understanding of assessment as an opportunity to explore potential sources of power available to clients via internal strengths, family resources, and neighborhood and community networks. We challenge students to reject the notion that they are to act as experts in response to clients who are to act as victims of psychic stress. In this way, we promote a practice grounded in collaboration and mutuality. We challenge students to critically examine and deconstruct the forms and relations of power and inequality that infiltrate both the clinical relationship and the broader social context in which they are embedded.

Employing pedagogical techniques grounded in critical and socioecological perspectives may be especially relevant for the social work profession, which consists of a primarily White workforce. As Olbert et al. (2018) noted, racial diagnostic disparities and misdiagnoses are "more extreme in studies with larger proportions of White individuals" (p.112). They recommended focusing on "primary prevention, reducing clinician bias, and improving awareness of the cultural mediation of psychotic symptom presentations" (Olbert et al., 2018, p. 112). Although contemporary events such as the COVID-19 pandemic and ongoing protests against police brutality have raised the work of antiracist scholars to the level of public consciousness, calls for integrating antiracism into social work curricula are not new (Abrams \& Moio, 2009; Basham, 2004; Nicotera, 2019). We echo antiracist scholars who reject the competency-based constraints of social work education and argue that "racial awareness is a formative process that involves cognitive, affective, and action-oriented changes that may not result from exposure to one or two courses" (Abrams \& Moio, 2009, p. 255). 
Figure 1. Diagnostic Decision-Making Process

Differential Diagnosis Decision-Making Process

Transdiagnostic Perspective

1

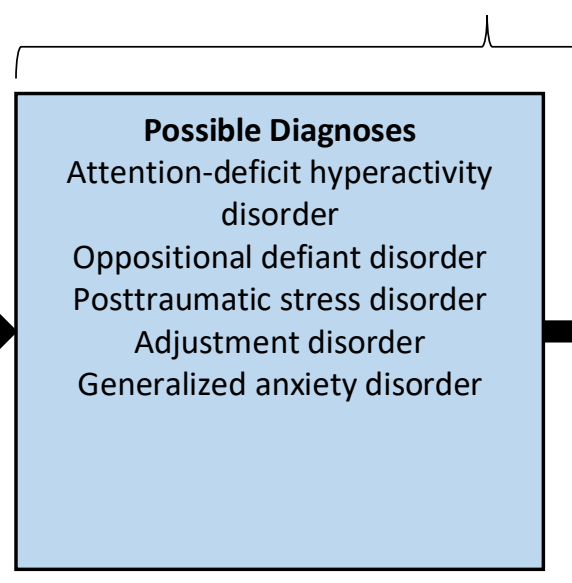

Critical and Socioecological Perspectives

\begin{tabular}{|c|}
\hline Shared Symptoms \\
Angry/irritable mood \\
Argumentative \\
behavior \\
Difficulty \\
concentrating \\
Not following \\
instructions \\
Psychomotor \\
agitation \\
\hline
\end{tabular}

1

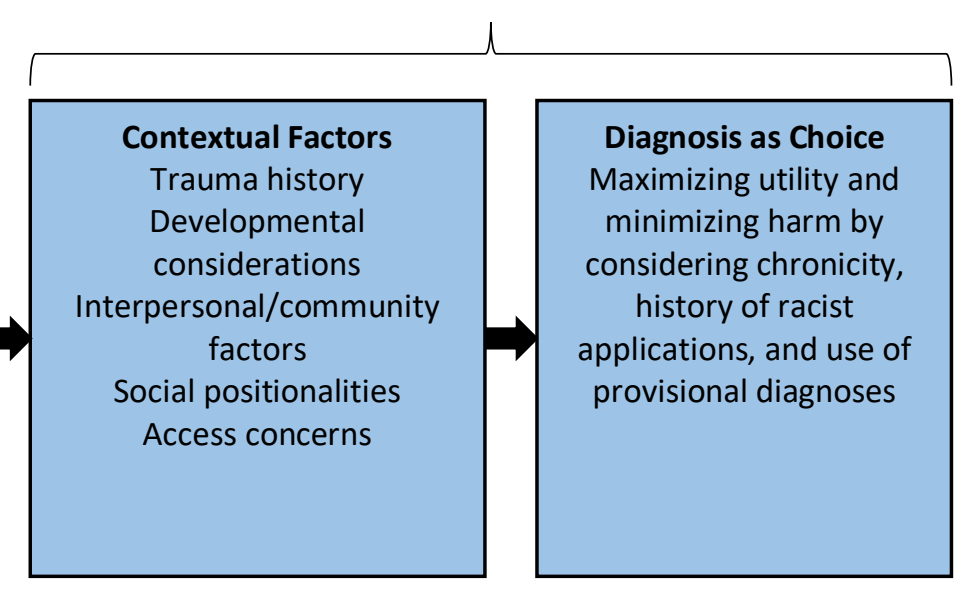

Note. Children exhibiting behavioral difficulties in school settings are commonly diagnosed with attention-deficit hyperactivity disorder or oppositional defiant disorder, but may be experiencing other psychological diagnoses such as anxiety disorders or trauma and stressor-related disorders. Applying a transdiagnostic perspective involves possible diagnoses that might explain presenting problems and then identifying

shared symptoms in order to determine which diagnoses most parsimoniously describe client symptomatology. Contextual factors-including children's trauma and developmental histories, social positionalities, and interpersonal and community variables that inform their school experiences - should ultimately inform the chosen diagnosis. Doing so ensures diagnostic decisions are informed by critical and socioecological perspectives. 
Teaching students to adopt transdiagnostic, critical, and socioecological perspectives does not involve teaching students a list diagnostic strategies per se; rather, it requires teaching students to think of diagnostics as a decision-making process wherein these perspectives can be used to maximize the utility of the DSM and minimize the potential for racial harm. This process is visually represented in Figure 1 using the example of a child exhibiting behavioral difficulties in a school setting. Choosing culturally responsive diagnoses requires providing students with an in-depth understanding of transdiagnostic symptomatology and positioning that understanding within critical and socioecological perspectives. By focusing on the development of these perspectives among our students, as well as competency in using the DSM, we attempt to make the formative process of racial awareness (Abrams \& Moio, 2009) explicit in our pedagogy.

\section{Reflexivity and Our Positionalities}

Encouraging critical self-reflection seems incomplete without discussing the impact of our positionalities on the formulation of this article. The first author identifies as a White, queer, cisgender man, and has been teaching BSW and MSW practice-oriented social work courses since 2015. The second author identifies as a Vietnamese, straight, cisgender woman whose teaching experiences have been situated in community practice settings focused on addressing racial and ethnic disparities in the criminal and juvenile justice systems. The third and fourth authors identify as White, straight, cisgender women, and have been teaching BSW and MSW practice-oriented social work courses since 2017. Our collaboration as scholars began while completing our doctoral studies together. We have relied on each other as research and practice consultants, peer debriefing team members, and teaching collaborators as we have developed our pedagogical and scholarly practices.

The conceptualizations and arguments presented in this article are primarily informed by our collective experiences of privilege - namely our White, straight, and cisgender identities. We also recognize that the ways in which we individually navigate the spaces we occupy are unique to our respective experiences and complex, intersectional identities. Our efforts to teach psychopathology using an antiracist pedagogy are rooted in our personal efforts to learn and implement antiracist frameworks in our respective scholarly activities. We have sought to integrate and center BIPOC perspectives, youth voices, and other marginalized identities in our research efforts, and clinical and community practices. In this article, we aimed to highlight theories and research from BIPOC scholars in the fields of psychopathology and antiracism, only to discover a dearth of BIPOC voices in the literature on psychopathology. This disparity seems to mirror our earlier discussion on issues of access and structural barriers that hinder the elevation or centering of BIPOC needs and perspectives.

Although any discussion of utilizing the DSM risks reifying White Supremacy in clinical social work practice, our hope is that this article represents an authentic effort to practice the critical self-reflection we encourage in our students by reflexively acknowledging the limits of our personal experiences. Our integration of transdiagnostic and critical perspectives in our teaching highlight one facet of our efforts to disrupt White Supremacy in social work practice. Until a new taxonomy is created to supplant the DSM- 
one rooted in antiracism - we will continue searching for frameworks and perspectives that pragmatically acknowledge the use of the DSM in the mental health field while actively critiquing the disparate harm it causes. We are committed to learning from individuals, scholars, and communities of color on how to respond to BIPOC who have been impacted by the misuse of the DSM.

\section{Benefits of Using a Transdiagnostic Perspective}

The benefits of teaching students from a transdiagnostic perspective include preparing social work students for a future shift in thinking about the classification of psychological disorders and its potential impact on clinical practice, bridging the gap between research and practice given the increasing focus among researchers on mechanistically transdiagnostic processes, and encouraging complex and critical thinking across socioecological levels. A transdiagnostic perspective allows social work students to avoid falling into a deficit-focused mindset of pathologizing human suffering. Rather, it allows them to conceptualize and contextualize the mechanisms and processes that initiate and maintain suffering, as well as those that promote wellbeing. We ultimately support the recommendation given by Sauer-Zavala et al. (2017) to focus on teaching mechanistically transdiagnostic processes as these can inform what they termed "shared mechanisms" treatments (p. 130). Mechanistically transdiagnostic processes are especially relevant to the topic of White supremacist applications of the DSM given the potentially transdiagnostic role of oppression and racial trauma in the development and maintenance of mental distress.

Employing a pedagogy that integrates transdiagnostic, critical, and socioecological perspectives also has specific benefits for disrupting White Supremacist applications of the DSM. These benefits include the consideration of racial trauma as a transdiagnostic process informing myriad disorders, as well as the conscious recognition of and decision to use fewer racially biased diagnoses when presented with such opportunities. For example, given the symptomatic overlap between the disorders described earlier (e.g., schizophrenia and mood disorders, oppositional defiant disorder and attention-deficit hyperactivity disorder), social workers can make a conscious choice to avoid reinforcing racist stereotypes by employing a critical lens during the differential diagnosis process. Additionally, employing culturally sensitive assessment techniques can help social workers evaluate the potentially traumatizing effects of oppression and marginalization, thereby positioning the etiology of their clients' suffering within deficit systems rather than within individuals.

\section{Conclusion}

The pedagogical approaches we have outlined in this article are rooted in our epistemic affinity for pragmatism, as well as our deep commitment to antiracist social work practice. Our recommendation to integrate transdiagnostic, critical, and socioecological perspectives is likely familiar to most social workers. They align with both the person-in-environment and biopsychosocial perspectives, which are foundational frameworks in social work education and practice. What we hope for, and have aimed to encourage in this article, is 
that social workers-practitioners, educators, and researchers alike-will apply the integration of these perspectives to the disruption of White Supremacy. Presently, social workers must learn the DSM in order to operate effectively in the mental health field, that is until a classification system is developed that balances contextualization, etiology, and antiracist principles. In the meantime, we believe employing the pedagogical approaches we have outlined in this article is one way to teach social work students to practice in the mental health field in a way that aligns with social work values and ethics.

\section{References}

Abrams, L. S., \& Moio, J. A. (2009). Critical Race Theory and the cultural competence dilemma in social work education. Journal of Social Work Education, 45(2), 245261. https://doi.org/10.5175/jswe.2009.200700109

Aggarwal, N. K., Rosenheck, R. A., Woods, S. W., \& Sernyak, M. J. (2012). Race and long-acting antipsychotic prescription at a community mental health center: A retrospective chart review. The Journal of Clinical Psychiatry, 73(4), 513-517. https://doi.org/10.4088/JCP.11m07161

Akinhanmi, M. O., Biernacka, J. M., Strakowski, S. M., McElroy, S. L., Balls Berry, J. E., Merikangas, K. R., Assari, S., McInnis, M. G., Schulze, T. G., LeBoyer, M., Tamminga, C., Patten, C., \& Frye, M. A. (2018). Racial disparities in bipolar disorder treatment and research: A call to action. Bipolar Disorders, 20(6), 506-514. https://doi.org/10.1111/bdi.12638

American Psychiatric Association. (2013). Diagnostic and statistical manual of mental disorders (5th ed.). Author.

Atteberry-Ash, B., Nicotera, N., \& Gonzales, B. (2019). Walk the talk of power, privilege, and oppression: A template analysis. Journal of Social Work Education, 57(1), 7-15. https://doi.org/10.1080/10437797.2019.1661917

Ballentine, K. L. (2019). Understanding racial differences in diagnosing ODD versus ADHD using Critical Race Theory. Families in Society, 100(3), 282-292. https://doi.org/10.1177/1044389419842765

Basham, K. (2004). Weaving a tapestry: Anti-racism and the pedagogy of clinical social work practice. Smith College Studies in Social Work, 74(2), 289-314. https://doi.org/10.1080/00377310409517717

Bedwell, J. S., Gooding, D. C., Chan, C. C., \& Trachik, B. J. (2014). Anhedonia in the age of RDoC. Schizophrenia Research, 160, 226-227. https://doi.org/10.1016/j.schres.2014.10.028

Bredström, A. (2019). Culture and context in mental health diagnosing: Scrutinizing the DSM-5 revision. Journal of Medical Humanities, 40(3), 347-363. https://doi.org/10.1007/s10912-017-9501-1

Burke, C. A. (2010). Mindfulness-based approaches with children and adolescents: A preliminary review of current research in an emergent field. Journal of Child and Family Studies, 19(2), 133-144. https://doi.org/10.1007/s10826-009-9282-x 
Carter, R. T. (2007). Racism and psychological and emotional injury: Recognizing and assessing race-based traumatic stress. The Counseling Psychologist, 35(1), 13-105. https://doi.org/10.1177/0011000006292033

Cerulo, K. A. (2010). Mining the intersections of cognitive sociology and neuroscience. Brain, Mind and Cultural Sociology, 38(2), 115-132. https://doi.org/10.1016/i.poetic.2009.11.005

Crenshaw, K. (1991). Mapping the margins: Intersectionality, identity politics, and violence against Women of Color. Stanford Law Review, 43(6), 1241-1299. https://doi.org/10.2307/1229039

Destoop, M., Morrens, M., Coppens, V., \& Dom, G. (2019). Addiction, anhedonia, and comorbid mood disorder: A narrative review. Frontiers in Psychiatry, 10(311), 1-14. https://doi.org/10.3389/fpsyt.2019.00311

Eack, S. M., Bahorik, A. L., Newhill, C. E., Neighbors, H. W., \& Davis, L. E. (2012). Interviewer-perceived honesty as a mediator of racial disparities in the diagnosis of schizophrenia. Psychiatric Services, 63(9), 875-880.

https://doi.org/10.1176/appi.ps.201100388

Evangelist, M., Ryan, J. P., Victor, B. G., Moore, A., \& Perron, B. E. (2017). Disparities at adjudication in the juvenile justice system: An examination of race, gender, and age. Social Work Research, 41(4), 199-212. https://doi.org/10.1093/swr/svx017

Fader, J. J., Kurlychek, M. C., \& Morgan, K. A. (2014). The color of juvenile justice: Racial disparities in dispositional decisions. Social Science Research, 44, 126-140. https://doi.org/10.1016/j.ssresearch.2013.11.006

Feisthamel, K. P., \& Schwartz, R. C. (2009). Differences in mental health counselors' diagnoses based on client race: An investigation of adjustment, childhood, and substance-related disorders. Journal of Mental Health Counseling, 31(1), 47-59. https://doi.org/10.17744/mehc.31.1.u82021637276wv1k

Fusar-Poli, P., Solmi, M., Brondino, N., Davies, C., Chae, C., Politi, P., Borgwardt, S., Lawrie, S. M., Parnas, J., \& McGuire, P. (2019). Transdiagnostic psychiatry: A systematic review. World Psychiatry, 18(2), 192-207. https://doi.org/10.1002/wps.20631

Gudiño, O. G., Martinez, J. I., \& Lau, A. S. (2012). Mental health service use by youths in contact with child welfare: Racial disparities by problem type. Psychiatric Services, 63(10), 1004-1010. https://doi.org/10.1176/appi.ps.201100427

Haight, W., Kayama, M., \& Gibson, P. A. (2016). Out-of-school suspensions of Black youths: Culture, ability, disability, gender, and perspective. Social Work, 61(3), 235243. https://doi.org/10.1093/sw/sww021

Helms, J. E., Nicolas, G., \& Green, C. E. (2012). Racism and ethnoviolence as trauma: Enhancing professional and research training. Traumatology: An International Journal, 18(1), 65-74. https://doi.org/10.1177/1534765610396728 
Hogg, M. A., Terry, D. J., \& White, K. M. (1995). A tale of two theories: A critical comparison of identity theory with social identity theory. Social Psychology Quarterly, 58(4), 255-269. https://doi.org/10.2307/2787127

Holmes, S. C., Facemire, V. C., \& DaFonseca, A. M. (2016). Expanding Criterion A for Posttraumatic Stress Disorder: Considering the deleterious impact of oppression. Traumatology, 22(4), 314-321. https://doi.org/10.1037/trm0000104

Kim, C. Y., Losen, D. J., \& Hewitt, D. T. (2010). The school-to-prison pipeline: Structuring legal reform. NYU Press.

Kim, M. (2014). Racial/ethnic disparities in depression and its theoretical perspectives. Psychiatric Quarterly, 85(1), 1-8. https://doi.org/10.1007/s11126-013-9265-3

Kourgiantakis, T., Sewell, K., McNeil, S., Logan, J., Lee, E., Adamson, K., McCormick, M., \& Kuehl, D. (2019). Social work education and training in mental health, addictions and suicide: A scoping review protocol. BMJ Open, 9, 1-6. https://doi.org/10.1136/bmjopen-2018-024659

La Roche, M. J., Fuentes, M. A., \& Hinton, D. (2015). A cultural examination of the DSM-5: Research and clinical implications for cultural minorities. Professional Psychology: Research and Practice, 46(3), 183-189. https://doi.org/10.1037/a0039278

Lyter, S. C., \& Lyter, L. L. (2012). Diagnostic and Statistical Manual of Mental Disorders: Making it work for social work. The International Journal of Interdisciplinary Social Sciences: Annual Review, 6, 53-64. https://doi.org/10.18848/1833-1882/cgp/v06i06/52093

Mallett, C. A. (2016). The school-to-prison pipeline: Disproportionate impact on vulnerable children and adolescents. Education and Urban Society, 49(6), 563-592. https://doi.org/10.1177/0013124516644053

Metzl, J. M. (2009). The protest psychosis: How schizophrenia became a Black disease. Boston, MA: Beacon Press.

Netherland, J., \& Hansen, H. B. (2016). The war on drugs that wasn't: Wasted Whiteness, "dirty doctors," and race in media coverage of prescription opioid misuse. Culture, Medicine and Psychiatry, 40(4), 664-686. https://doi.org/10.1007/s11013016-9496-5

Nicotera, A. (2019). Social justice and social work, a fierce urgency: Recommendations for social work social justice pedagogy. Journal of Social Work Education, 55(3), 460-475. https://doi.org/10.1080/10437797.2019.1600443

Olbert, C. M., Nagendra, A., \& Buck, B. (2018). Meta-analysis of Black vs. White racial disparity in schizophrenia diagnosis in the United States: Do structured assessments attenuate racial disparities? Journal of Abnormal Psychology, 127(1), 104-115. https://doi.org/10.1037/abn0000309

Probst, B. (2013). "Walking the tightrope:" Clinical social workers' use of diagnostic and environmental perspectives. Clinical Social Work Journal, 41(2), 184-191. 
https://doi.org/10.1007/s10615-012-0394-1

Riboni, F., \& Belzung, C. (2017). Stress and psychiatric disorders: From categorical to dimensional approaches. Current Opinion in Behavioral Sciences, 14, 72-77. https://doi.org/10.1016/j.cobeha.2016.12.011

Rodriguez-Seijas, C., Eaton, N. R., \& Krueger, R. F. (2015). How transdiagnostic factors of personality and psychopathology can inform clinical assessment and intervention. Journal of Personality Assessment, 97(5), 425-435. https://doi.org/10.1080/00223891.2015.1055752

Sauer-Zavala, S., Gutner, C. A., Farchione, T. J., Boettcher, H. T., Bullis, J. R., \& Barlow, D. H. (2017). Current definitions of "transdiagnostic" in treatment development: A search for consensus. Behavior Therapy, 48(1), 128-138. https://doi.org/10.1016/j.beth.2016.09.004

Schwartz, R. C., \& Blankenship, D. M. (2014). Racial disparities in psychotic disorder diagnosis: A review of empirical literature. World Journal of Psychiatry, 4(4), 133140. PubMed. https://doi.org/10.5498/wjp.v4.i4.133

Simpson, S. M., Krishnan, L. L., Kunik, M. E., \& Ruiz, P. (2007). Racial disparities in diagnosis and treatment of depression: A literature review. Psychiatric Quarterly, 78(1), 3-14. https://doi.org/10.1007/s11126-006-9022-y

Tonry, M. (1994). Race and the war on drugs. University of Chicago Legal Forum, 1(4), $25-81$. https://chicagounbound.uchicago.edu/cgi/viewcontent.cgi?article=1155\&context=uclf

Tsai, A. C., Kiang, M. V., Barnett, M. L., Beletsky, L., Keyes, K. M., McGinty, E. E., Smith, L. R., Strathdee, S. A., Wakeman, S. E., \& Venkataramani, A. S. (2019). Stigma as a fundamental hindrance to the United States opioid overdose crisis response. PLoS Medicine, 16(11), 1-18. https://doi.org/10.1371/journal.pmed.1002969

Visser, S. N., Deubler, E. L., Bitsko, R. H., Holbrook, J. R., \& Danielson, M. L. (2016). Demographic differences among a national sample of US youth with behavioral disorders. Clinical Pediatrics, 55(14), 1358-1362. https://doi.org/10.1177/0009922815623229

Whooley, O. (2014). Nosological reflections: The failure of DSM-5, the emergence of RDoC, and the decontextualization of mental distress. Society and Mental Health, 4(2), 92-110. https://doi.org/10.1177/2156869313519114

Author note: Address correspondence to Michael R. Riquino, School of Social Welfare, University of Kansas, Twente Hall, 1545 Lilac Lance, Lawrence, KS 66045. Email: mriquino@ku.edu 